\title{
The Use of Hinged External Fixation to Provide Additional Stabilization for Fractures of the Distal Humerus
}

\author{
Christopher R. Deuel, MS, Philip Wolinsky, MD, Eric Shepherd, MD, \\ and Scott J. Hazelwood, PhD
}

Objective: To assess improvements in fixation stability when a hinged unilateral external fixator is used to supplement compromised internal fixation for distal humerus fractures.

Methods: Removing a 1-cm section of the distal humerus in cadaveric whole-arm specimens created a comminuted distal humerus fracture model (AO type 13-A3). Fixation was then performed using different constructs representing optimal, compromised, or supplemented internal fixation. Internal fixation consisted of either 2 reconstruction plates with 1, 2, or 3 (optimal) distal attachment screws, or crossing medial and lateral cortical screws. A hinged external fixator was applied in combination with compromised internal fixation. The stability of the different constructs was then evaluated using 3-point bending stiffness and distal fragment displacement measurements during flexion and extension testing.

Results: Addition of the external fixator increased the stiffness of all constructs. Stiffness of the compromised reconstruction plate constructs with supplemented fixation was similar to or significantly greater than that of optimal internal fixation. Addition of the fixator to the reconstruction plates with 1 screw or the crossing screws produced displacements of the distal fragment that were similar to those of the compromised constructs alone. However, medial/lateral and anterior/posterior displacements of the distal fragment during flexion and extension of the elbow for supplemented fixation were found to be greater than those for optimal internal fixation.

Conclusions: The use of a hinged external fixator for supplemental fixation of distal humerus fractures may be effective in cases where internal fixation is severely compromised, although displacements may increase above optimal fixation.

Key Words: hinged external fixation, distal humerus fractures, supracondylar humerus fixation, internal fixation, biomechanics

\section{INTRODUCTION}

The current standard of care for fixation of supracondylar fractures of the humerus in adults is open reduction and internal fixation. ${ }^{1-5}$ Two plates usually are used with 1 placed on the medial column and the second on the lateral column of the distal humerus to provide optimal internal fixation. ${ }^{6-8}$ It is recommended that each plate have a minimum of 3 screws inserted proximal as well as distal to the fracture site to provide rigid fixation. Obtaining sufficient distal fixation can be challenging, particularly in the elderly, because of osteoporosis or comminuted fracture patterns. ${ }^{1-5}$ The proximity of the fracture to the elbow joint and limited amount of bone available for fixation in the distal fragment may not allow for placement of all 3 distal screws in each column plate, potentially leading to a decrease in stiffness of the construct and an increase in relative displacement of the bone fragments. ${ }^{9}$ Failure usually occurs through loss of fixation in the distal fragment. ${ }^{5,9}$ Between $2 \%$ and $10 \%$ delayed unions or nonunions of fractures of the distal humerus have been observed following internal fixation. ${ }^{10}$ Ali et al found that $75 \%$ of referred patients with nonunion had inadequate primary fixation consisting of either screws, wires, or plates with a limited number of screws in the proximal or distal fragments. ${ }^{11}$ Clearly, obtaining initial rigid fracture fixation is critical in achieving union of the bony fragments for fractures of the distal humerus. ${ }^{10-12}$

Early rehabilitation is also essential in restoring proper function of the elbow joint. ${ }^{13,14}$ Because of this, at our institution and others, hinged external fixators have been used for the past few years to treat elbow injuries, including fracture dislocations and radial head fractures, and after elbow contracture releases. ${ }^{15-18}$ Hinged external fixators allow early range of motion of the elbow while protecting the repairs of the ligamentous and/or bony injuries about the elbow joint, but their effects on stability of fractures in close proximity to the joint have yet to be examined experimentally. Recent case studies have demonstrated promising results. ${ }^{19,20}$ We hypothesized that a hinged elbow external fixator could be used to supplement internal fixation of the distal humerus in cases where optimal internal fixation cannot be achieved. This would allow for early rehabilitation while potentially increasing the stability of compromised internal fixation. The purpose of this study was to determine if a new hinged unilateral external fixation device, when used in combination with compromised internal fixation, would result in increased construct stiffness and fracture stability. 


\section{MATERIALS AND METHODS}

Fifteen fresh frozen cadaveric right upper limbs disarticulated at the shoulder joint were used for this study. Donors with a known history of metabolic bone disease were excluded. The skin was excised proximal to the wrist, and all forearm muscles and soft tissues were left intact, as were the elbow joint capsule and ligaments. All soft tissues were removed from the proximal and middle third of the humerus. A 1-cm section of the distal humerus was removed $2 \mathrm{~cm}$ proximal to the center of the olecranon fossa to simulate a comminuted supracondylar fracture of the humerus (AO type 13-A3) in which there is no cortical contact between fragments (Fig. 1). This was considered a worst-case situation in terms of stability of the fracture. Internal fixation consisting of either two 3.5-mm reconstruction plates (Synthes, Paoli, Pennsylvania) or crossing medial and lateral 3.5-mm cortical screws (Synthes) was then applied to the fracture while maintaining a 1-cm gap. The crossing screws were placed across the fracture to model a construct with minimal stability, and a lag technique was not used. The 2 reconstruction plates were contoured to the distal humerus and placed at 90 degrees to each other on the medial column and posterior aspect of the lateral column. A 9-hole plate was used medially, and a 10-hole plate was placed posterolaterally. Three 3.5-mm cortical screws (Synthes) were used to attach each plate proximal to the fracture site with 1.0-, 2.0-, 3.0-, or 4.0-mm cancellous screws (Synthes) used to attach each plate to the distal fragment. Bicortical purchase was achieved for all specimens, with screw length determined using a depth gauge. Three screws were secured distally in each plate to represent optimal internal fixation (OF), and 1 or 2 screws were secured distally in each plate to represent compromised internal fixation.

To examine the effects on stability of compromised fixation, a unilateral hinged external fixator (EBI, Parsippany, New Jersey) was applied to the lateral side of the extremity according to the instructions supplied by the manufacturer to supplement the suboptimal fixation. First, an axis wire was placed under direct vision from lateral to medial across the distal humerus using the condyles as landmarks. The fixator with its guide was then placed on the wire, and two $6.0-\mathrm{mm}$ tapered cortical bone screws were placed in the humeral shaft.

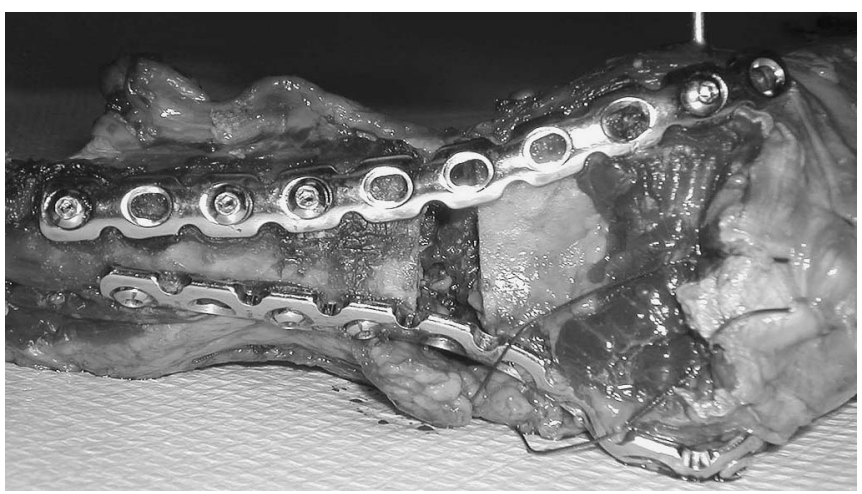

FIGURE 1. Cadaveric specimen with 2 plates placed at 90 degrees along the medial and lateral column with a $1-\mathrm{cm}$ gap between the proximal and distal segments of the humerus.
Two 4.5-mm tapered cortical bone screws were then added to the ulna shaft. Surgeries for all constructs were performed by an experienced attending surgeon.

A total of 7 fixation constructs and 15 test specimens were tested in 3-point bending stiffness and flexion/extension tests. One set of 5 specimens (Group A) was tested with 2 reconstruction plates having 1,2 , or $3 \operatorname{screw}(s)$ in the distal fragment, and a second set of 5 specimens (Group B) was tested with the external fixator and reconstruction plates having 1 or 2 screw(s) distally. A third set of 5 specimens (Group C) was tested with crossing cortical screws, with and without the external fixator. An overview of the treatments and test specimens is given in Table 1.

\section{Mechanical Testing}

Testing was performed to evaluate the effects of the external fixator on enhancing the stability of constructs representing compromised internal fixation. Stability was evaluated using 2 different experimental methods. One method involved measuring stiffness of the constructs across the fracture in bending, which previously has been used in comparisons of stability between different fracture fixation constructs. ${ }^{6,7,21}$ For the second experiment, displacements of the distal fragment were measured using an electromagnetic tracking device during flexion and extension of the elbow. Similar electromagnetic tracking devices have been used in cadaveric studies of elbow kinematics and stability. ${ }^{22-24}$ Our goal was to determine if external fixation increased the bending stiffness of the compromised fixation and reduced displacements of the distal fragment during motion of the elbow, thus enhancing the stability of compromised internal fixation for fractures of the distal humerus.

\section{Stiffness}

Stiffness of the constructs was measured in 3-point anterior/posterior bending using an Instron 1122 Material Testing Machine (Instron Corporation, Norwood, Massachusetts). The humerus was positioned horizontally and supported posteriorly on either side of the osteotomy by an L-bracket and a custom-made support that conformed to the olecranon fossa (Fig. 2). A linear, vertical load from 0 to $500 \mathrm{~N}$ was applied to the anterior surface of the humerus approximately $89 \mathrm{~mm}$ proximal to the center of the olecranon fossa support at

\begin{tabular}{lcccc}
\hline TABLE 1. Study Design Table \\
\hline Treatment & $\begin{array}{c}\text { Proximal } \\
\text { Screws }\end{array}$ & $\begin{array}{c}\text { Distal } \\
\text { Screws }\end{array}$ & Ex-Fix & $\begin{array}{c}\text { Specimen } \\
\text { Group }\end{array}$ \\
\hline OF & 3 & 3 & No & A \\
2DS & 3 & 2 & No & A \\
1DS & 3 & 1 & No & A \\
2DSX & 3 & 2 & Yes & B \\
1DSX & 3 & 1 & Yes & B \\
CS & NA & NA & No & C \\
CSX & NA & NA & Yes & C
\end{tabular}

OF, optimal fixation; 2DS, reconstruction plates with 2 distal screws; 1DS, reconstruction plates with 1 distal screw; 2DSX, 2DS + fixator; 1DSX, 1DS + fixator; $\mathrm{CS}$, crossing screws; CSX, CS + fixator. 


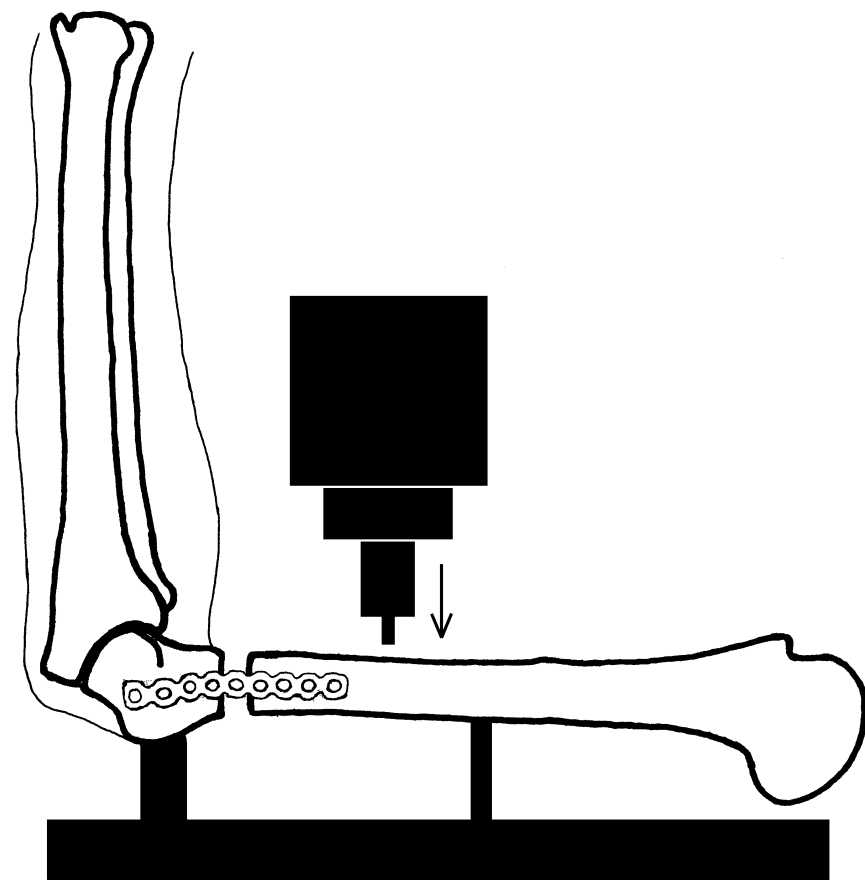

FIGURE 2. Schematic of fixture used for stiffness testing with the cadaveric specimen being loaded in 3-point bending. The posterior supports were $127 \mathrm{~mm}$ apart, and loading was applied to the anterior surface $59 \mathrm{~mm}$ proximal to the edge of the fracture and $38 \mathrm{~mm}$ from the proximal support.

a rate of $2 \mathrm{~mm} / \mathrm{min}$. This resulted in a maximum moment of 4.5 Nm applied at the fracture site. ${ }^{7}$ Testing was stopped when a load of $500 \mathrm{~N}$ was reached. Loading rates and magnitudes were chosen to allow measurements of stiffness within the linear elastic range of the construct while preventing plastic deformation. The elbow remained flexed approximately 90 degrees during testing. The load and linear displacement at the crosshead of the Instron were collected at a rate of $200 \mathrm{~Hz}$. Stiffness of the construct was defined as the slope of the best-fit linear regression curve of the load versus displacement data. Each construct was tested on 5 different arms, and an average stiffness value for the construct was calculated.

\section{Flexion and Extension}

Flexion and extension testing was performed to measure displacements of the distal humerus fragment resulting from instability of the constructs during rotation of the elbow. A custom aluminum fixture was built to rigidly hold the humerus, and position measurements of the distal humerus fragment were taken at $15 \mathrm{~Hz}$ using an electromagnetic tracking device (Flock of Birds, Ascension Technology Corporation, Milton, Vermont) with a positional resolution of $0.25 \mathrm{~mm}$. The device consists of a transmitter that sends a pulsed magnetic field and a small receiver that senses its intensity. The position of the receiver then may be determined relative to the transmitter based on the intensity of the magnetic field. A more complete description of the system may be found in Bottlang et al. ${ }^{25}$

The receiver was attached to the distal fragment of the humerus medially using a small plate and pin that passed through the epicondyles of the distal humerus. The receiver measured displacement of the distal fragment relative to the transmitter, which was fixed with respect to the proximal humerus (Fig. 3) during either flexion or extension of the elbow. Weights were suspended from sutures attached to the biceps $(10 \mathrm{~N})$, brachialis $(10 \mathrm{~N})$, and triceps $(2 \mathrm{~N})$ tendons to simulate muscle forces acting across the joint in flexion. ${ }^{26}$ A cable wound around a spool driven by a DC motor was attached to the anterior surface of the ulna approximately $150 \mathrm{~mm}$ from the elbow joint center of rotation. The DC motor was positioned remotely so that electromagnetic interference would not create an error in the position measurements. The arm was initially placed in extension ( 0 degrees), then rotated to a flexed position (90 degrees) at 30 degrees/sec using the DC motor while displacement data of the distal fragment were collected. Next, weights were suspended from sutures attached to the biceps $(1 \mathrm{~N})$, brachialis $(1 \mathrm{~N})$, and triceps $(20 \mathrm{~N})$ tendons to simulate muscle forces acting across the joint in extension. ${ }^{26}$ With the arm positioned at 90 degrees, the direction of the motor was reversed to allow the arm to extend at 30 degrees/sec while displacement data of the distal fragment were collected. The forearm was placed in a supinated position for both flexion and extension testing. Both flexion and extension tests were repeated 3 times, and data from the third test were used for the analyses. Each construct was tested on 5 different arms, and average maximum displacements

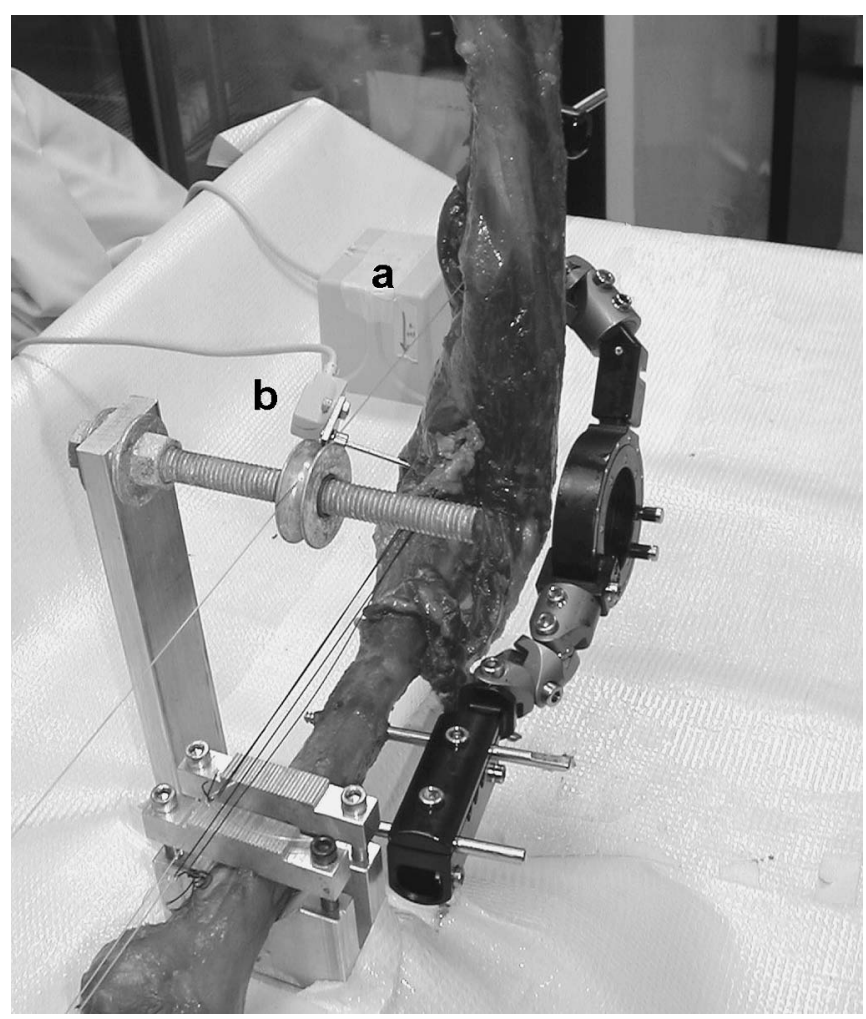

FIGURE 3. Flexion and extension test fixture with the cadaveric specimen showing the Flock of Birds transmitter (A) and receiver (B) used to measure displacement of the distal fragment. 
for proximal/distal (P/D), medial/lateral $(\mathrm{M} / \mathrm{L})$, and anterior/ posterior $(\mathrm{A} / \mathrm{P})$ motion were determined for the construct.

\section{Statistical Analysis}

A 1-way analysis of variance (ANOVA) was performed using SAS Software (SAS Institute, Cary, North Carolina) to determine if there were statistically significant differences in stiffness values and fragment displacements between constructs $(P<0.05$ significant). It was necessary to perform a $\log$ transformation of the displacement data prior to the analyses to satisfy the assumption of normal distribution of the residual error and constant residual variance. A pairwise post hoc analysis was performed, when appropriate, using a Dunnett-Hsu test to detect differences in stiffness or fracture displacement for all constructs using optimal internal fixation as the control. Additionally, all other pairwise comparisons were performed using a Tukey-Kramer test to determine which fixation constructs exhibited differences in stiffness or fracture displacement.

\section{RESULTS}

\section{Stiffness}

Whereas stiffness of the plates with 1 distal screw (1DS) was $23 \%$ less than optimal internal fixation $(P=0.0014)$, addition of the hinged external fixator to the compromised construct (1DSX) significantly increased the stiffness by $42 \%$ $(P=0.0133$; Fig. 4), resulting in a value statistically equivalent to that of the optimal construct. The stiffness of the reconstruction plates with 2 distal screws (2DS) also was statistically similar to optimal fixation. Addition of the fixator to the plates with 2 distal screws (2DSX) significantly increased the stiffness $40 \%$ greater than that of $2 \mathrm{DS}(P=0.0040)$ and $26 \%$ greater than that of OF $(P=0.0358)$. The crossing cortical screws alone (CS) were the least stiff fixation method examined. Addition of the external fixator more than doubled the stiffness of the CS construct, although this was still significantly less than optimal internal fixation $(P=0.0036)$.

\section{Flexion and Extension}

Displacements of the distal fragment were similar for 2DS compared with optimal fixation in both flexion (Fig. 5A) and extension (Fig. 5B), whereas the 1DS construct exhibited significantly larger displacements in the $\mathrm{M} / \mathrm{L}$ direction in flexion $(P=0.0320)$ and the $\mathrm{P} / \mathrm{D}$ and $\mathrm{M} / \mathrm{L}$ directions in extension $(P=0.0214$ and 0.0412 , respectively). Displacements for the 1DS and 2DS constructs were statistically similar for all directions.

Addition of the fixator to the compromised reconstruction plate constructs (1DSX and 2DSX) increased the displacements relative to $\mathrm{OF}$ in all directions $(P=0.0014$ to $0.0400)$ with the exception of P/D for the 2DSX construct (Figs. 5A and 5B). Also, the A/P displacements for 1DSX ( $P=$ $0.0176)$ and 2DSX $(P=0.0123)$ in flexion and the $\mathrm{P} / \mathrm{D}$ displacements for 1DSX in both flexion $(P=0.0343)$ and extension $(P=0.0148)$ were significantly greater than the respective $2 \mathrm{DS}$ measurements.

The displacements of the CS construct were statistically greater than those for $\mathrm{OF}$ in all directions $(P=0.0009$ to

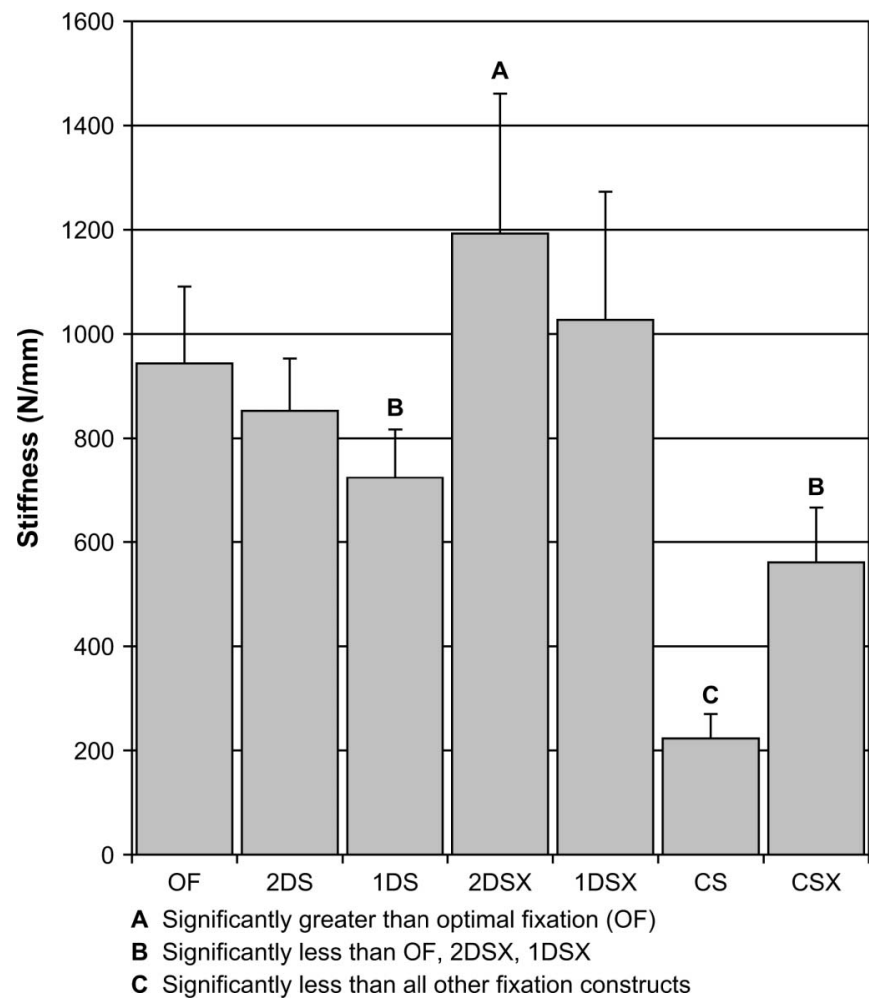

FIGURE 4. Three-point bending stiffness $(\mathrm{N} / \mathrm{mm})$ of all constructs examined for the fixation of the distal humerus.

0.0174) except for P/D when tested in flexion (Figs. 5A and 5B). Supplementation of the crossing screws with the fixator (CSX) resulted in displacements significantly greater than those for OF $(P<0.001)$ and 2DS $(P<0.05)$ for all directions tested.

\section{DISCUSSION}

Internal fixation of fractures of the distal humerus can be challenging, particularly when the fracture line or comminution does not allow for placement of at least 3 screws distal to the fracture site. ${ }^{8,9}$ Alternative treatments that have been suggested for these cases include total elbow arthroplasty for older patients or nonoperative treatment- the so-called "bag of bones" usually used for individuals who are older and low demand. ${ }^{8,9,27,28}$ In the study presented here, we hypothesized that a hinged elbow external fixator may be used to enhance the stability of compromised internal fixation in these cases. Our results demonstrate that the addition of a hinged external fixator to compromised internal fixation is effective in increasing the stiffness of the constructs in a cadaveric model but with the risk of possibly increasing motion at the fracture site.

Our study had several strengths, including the use of a cadaveric model with an intact joint capsule and simulated muscle loading applied to the muscle tendons. Precise measurements of displacement of the distal humerus bone fragment were taken. Additionally, to the best of our knowledge there are currently no published experimental studies 

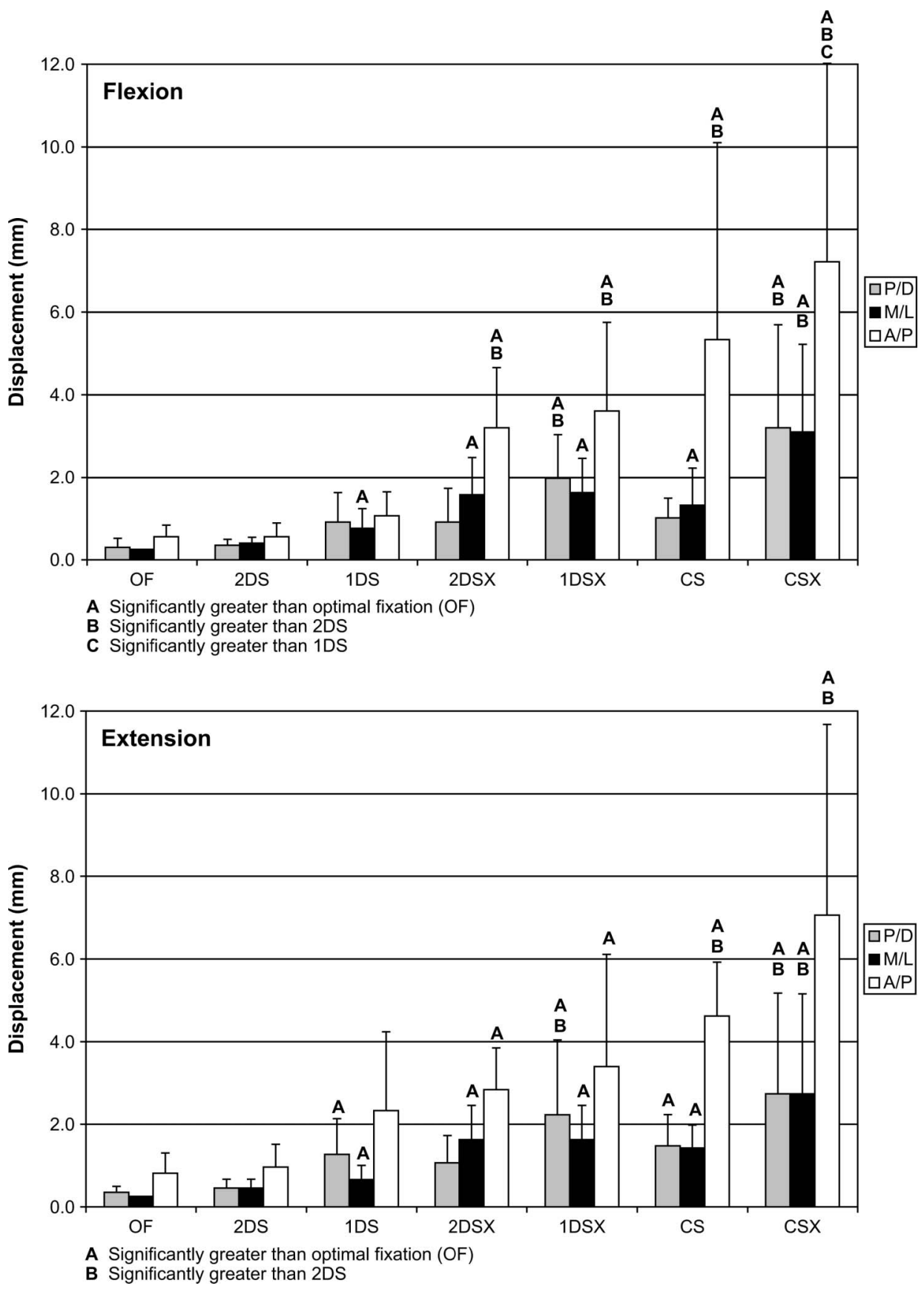

FIGURE 5. A, Displacements of the distal fragment $(\mathrm{mm})$ for all constructs during flexion of the elbow. $\mathrm{B}$, Displacements of the distal fragment $(\mathrm{mm})$ for all constructs during extension of the elbow.

evaluating the supplementation of less than optimal internal fixation or comparing compromised internal fixation against optimal internal fixation for fractures of the distal humerus. Helfet and Hotchkiss compared different methods of internal fixation of the distal humerus but did not consider compromised internal fixation. ${ }^{6}$ Korner et al compared locking compression plates (LCPs) against conventional reconstruction plates for distal humerus fracture fixation. ${ }^{7}$ No significant differences in stiffness or strength were found by Korner et al when the 2 different types of plates were used in the same configuration; however, 3 distal screws were always placed in each plate and compromised internal fixation was not considered. ${ }^{7}$ Because LCPs are designed to increase primary stability, an interesting variation of their study would be to remove 1 or 2 screws distally and repeat the test procedures, as was done in our study.

Although attempts were made to limit variability in testing conditions between samples and maintain physiologic loading, there were several limitations to the study. Cadaveric specimens were used exclusively to model the dynamics of the elbow joint, with testing performed at room temperature in a nonphysiologic environment. During the course of testing, 
1 specimen was replaced because of poor bone quality as evidenced by screw loosening. Another limitation was that constant loads were applied to the biceps, triceps, and brachialis tendons during flexion and extension testing, although it is known that muscle forces vary during motion of the joint. ${ }^{29}$ Additionally, flexion and extension of the elbow required applying a force to the mid-diaphysis of the ulna, creating a nonphysiologic loading condition.

Despite these limitations, we found that there was no significant difference in stiffness or distal fragment displacements between the constructs with 2 and 3 distal screws (2DS, $\mathrm{OF}$ ), suggesting that only 2 screws are needed distal to the fractures site to stabilize these fractures during 3-point bending or flexion/extension testing. However, removal or loosening of screws in the distal fragment so that only 1 screw remains secure would result in a construct (1DS) significantly less stable than optimal fixation, increasing the risk for delayed or nonunion of the bone. The addition of an external fixator significantly increased the stiffness of all constructs. Although the group with 1 distal screw and no fixator (1DS) was significantly less stiff than OF, adding the fixator enhanced the stiffness of the construct to the point where the 2 groups were similar. Using the fixator with the more severely compromised constructs (1DSX, CSX) increased the stiffness of fixation without significantly increasing motion of the distal fragment relative to that of internal fixation only (1DS, CS). Although our results suggest that supplementation with the fixator would not be necessary for 2DS, displacements of the distal fragment significantly increased when the fixator was used to supplement the reconstruction plates with 2 distal screws (2DSX) despite our finding that stiffness was increased. Motion of the distal fragment with external fixation was greater than that of optimal fixation in most cases. Based on the results of Madey et al, increases in displacement of the distal fracture fragment may have been the result of slight misalignment of the axis of the fixator with the true axis of elbow rotation, resulting in increased friction between the distal fragment and joint surface. ${ }^{22}$ Proper alignment of the fixator hinge axis of rotation with the elbow's anatomic axis of rotation may be a critical factor in limiting the displacement of the distal fragment during flexion and extension testing of the elbow. Nonphysiologic loading conditions and bending of the humerus with the elbow in extension as a result of the weight of the fixator also may have been a factor in increased displacement measurements of the distal fragment.

Care must be taken when making clinical recommendations based on the results of a mechanical study. However, test results may be useful when considering fracture fixation implants. The results of this study suggest that it is possible to increase the stability of the construct through the supplemental use of a hinged external fixator in cases of severely compromised internal fixation, although displacements of the distal fragment may increase above those for optimal internal fixation during flexion and extension of the elbow.

\section{ACKNOWLEDGMENTS}

This research was supported in part by a gift from EBI and hardware provided by EBI and Synthes. The authors would like to gratefully thank Alan Kwan for his assistance with mechanical testing and Dr Neil Willits for his assistance with the statistical analyses.

\section{REFERENCES}

1. Huang TL, Chiu FY, Chuang TY, et al. The results of open reduction and internal fixation in elderly patients with severe fractures of the distal humerus: a critical analysis of the results. J Trauma. 2005;58: 62-69.

2. Kinik H, Atalar H, Mergen E. Management of distal humerus fractures in adults. Arch Orthop Trauma Surg. 1999;119:467-469.

3. Srinivasan K, Agarwal M, Matthews SJ, et al. Fractures of the distal humerus in the elderly: is internal fixation the treatment of choice? Clin Orthop Relat Res. 2005;434:222-230.

4. Pereles TR, Koval KJ, Gallagher M, et al. Open reduction and internal fixation of the distal humerus: functional outcome in the elderly. J Trauma. 1997;43:578-584.

5. Korner J, Lill H, Muller LP, et al. Distal humerus fractures in elderly patients: results after open reduction and internal fixation. Osteoporos Int 2005;16(Suppl 2):S73-79.

6. Helfet DL, Hotchkiss RN. Internal fixation of the distal humerus: a biomechanical comparison of methods. J Orthop Trauma. 1990;4: 260-264.

7. Korner J, Diederichs G, Arzdorf M, et al. A biomechanical evaluation of methods of distal humerus fracture fixation using locking compression plates versus conventional reconstruction plates. J Orthop Trauma. 2004 18:286-293.

8. Molloy S, Jasper LE, Burkhart BG, et al. Interference Kirschner wires augment distal humeral fracture fixation in the elderly. J Orthop Trauma. 2005;19:377-379.

9. O'Driscoll SW. Optimizing stability in distal humeral fracture fixation. $J$ Shoulder Elbow Surg. 2005;14:186S-194S.

10. Helfet DL, Kloen P, Anand N, et al. Open reduction and internal fixation of delayed unions and nonunions of fractures of the distal part of the humerus. J Bone Joint Surg Am. 2003;85-A:33-40.

11. Ali A, Douglas H, Stanley D. Revision surgery for nonunion after early failure of fixation of fractures of the distal humerus. J Bone Joint Surg Br. 2005;87-B:1107-1110.

12. Horne G. Supracondylar fractures of the humerus in adults. J Trauma. 1980;20:71-74.

13. Aitken GK, Rorabeck CH. Distal humeral fractures in the adult. Clin Orthop Relat Res. 1986;207:191-197.

14. Browne AO, O'Riordan M, Quinlan W. Supracondylar fractures of the humerus in adults. Injury. 1986;17:184-186.

15. McKee MD, Pugh DM, Wild LM, et al. Standard surgical protocol to treat elbow dislocations with radial head and coronoid fractures. Surgical technique. J Bone Joint Surg Am. 2005;87-A(Suppl 1):22-32.

16. Jupiter JB, Ring D. Treatment of unreduced elbow dislocations with hinged external fixation. J Bone Joint Surg Am. 2002;84-A: 1630-1635.

17. Ring D, Hotchkiss RN, Guss D, et al. Hinged elbow external fixation for severe elbow contracture. J Bone Joint Surg Am. 2005;87-A: 1293-1296.

18. Stavlas P, Gliatis J, Polyzois V, et al. Unilateral hinged external fixator of the elbow in complex elbow injuries. Injury. 2004;35:1158-1166.

19. Haasper C, Jagodzinski M, Krettek C, et al. Hinged external fixation and closed reduction for distal humerus fracture. Arch Orthop Trauma Surg. 2006;126:188-191.

20. Hall J, Schemitsch EH, McKee MD. Use of a hinged external fixator for elbow instability after severe distal humeral fracture. J Orthop Trauma. 2000; $14: 442-445$.

21. Schemitsch EH, Tencer AF, Henley MB. Biomechanical evaluation of methods of internal fixation of the distal humerus. $J$ Orthop Trauma. 1994;8:468-475.

22. Madey SM, Bottlang M, Steyers CM, et al. Hinged external fixation of the elbow: optimal axis alignment to minimize motion resistance. J Orthop Trauma. 2000;14:41-47.

23. King GJ, Itoi E, Niebur GL, et al. Motion and laxity of the capitellocondylar total elbow prosthesis. J Bone Joint Surg Am. 1994; 76-A:1000-1008. 
24. O’Driscoll SW, Morrey BF, Korinek S, et al. Elbow subluxation and dislocation. A spectrum of instability. Clin Orthop Relat Res. 1992;280:186-197.

25. Bottlang M, Marsh JL, Brown TD. Factors influencing accuracy of screw displacement axis detection with a D.C.-based electromagnetic tracking system. J Biomech Eng. 1998;120:431-435.

26. Bottlang M, Madey SM, Steyers CM, et al. Assessment of elbow joint kinematics in passive motion by electromagnetic motion tracking. J Orthop Res. 2000;18:195-202.
27. Kamineni S, Morrey BF. Distal humeral fractures treated with noncustom total elbow replacement. J Bone Joint Surg Am. 2004;86-A: 940-947.

28. Kamineni S, Morrey BF. Distal humeral fractures treated with noncustom total elbow replacement. Surgical technique. J Bone Joint Surg Am. 2005; 87-A(Suppl 1):41-50.

29. Murray WM, Delp SL, Buchanan TS. Variation of muscle moment arms with elbow and forearm position. J Biomech. 1995;28:513-525. 